\begin{tabular}{|c|l|}
\hline Title & Colloidal cry stallization utilizing interfaces of unidirectionally growing ice crystals \\
\hline Author(s) & Suzuki, Y oshihisa; Sazaki, Gen; Hashimoto, Kaori; Fujiwara, Takahisa; Furukawa, Y oshinori \\
\hline Citation & $\begin{array}{l}\text { Journal of crystal growth, 383, 67-71 } \\
\text { https://doi.org/10.1016/.jrysgro.2013.08.026 }\end{array}$ \\
\hline Issue Date & 2013 11-15 \\
\hline Doc URL & http://hdl.handle.net/2115/54119 \\
\hline Type & article (author version) \\
\hline File Information & JCG38367-71.pdf \\
\hline
\end{tabular}

Instructions for use 


\title{
Colloidal crystallization utilizing interfaces of unidirectionally growing ice crystals
}

\author{
Yoshihisa Suzuki ${ }^{1, *}$, Gen Sazaki ${ }^{2}$, Kaori Hashimoto ${ }^{3}$, Takahisa Fujiwara ${ }^{3}$, Yoshinori \\ Furukawa $^{2}$
}

1 Institute of Technology and Science, The University of Tokushima, 2-1

Minamijosanjima, Tokushima 770-8506, JAPAN

${ }^{2}$ Institute of Low Temperature Science, Hokkaido University, N19-W8 Kita-ku, Sapporo 060-0819, JAPAN

${ }^{3}$ Graduate School of Advanced Technology and Science, The University of Tokushima, 2-1 Minamijosanjima, Tokushima 770-8506, JAPAN

\footnotetext{
* Corresponding author. Tel.: +81 88656 7415; Fax: +81 886557025 E-mail address: suzuki@chem.tokushima-u.ac.jp (Y.Suzuki).
}

\begin{abstract}
We show the possibility of unidirectional freezing of colloidal random suspensions as a novel method for growing colloidal crystals. First we confirmed the rejection of polystyrene particles from unidirectionally growing ice-water interfaces by observing color gradations of colloidal crystals formed in front of the interfaces. The rejection of particles
\end{abstract}


from the growing interfaces increased particle concentration in the colloidal crystals. Then we succeeded in achieving colloidal crystallization in front of ice-water interfaces during the unidirectional freezing of water suspensions of polystyrene particles. The colloidal crystals thus obtained exhibited a columnar shape that is typically observed in the unidirectional colloidal crystallization using centrifugation.

\section{Keywords}

A1. Directional solidification, A1. Growth models, A1. Nucleation, B1. Nanomaterials, B2. Nonlinear optic materials

\section{Introduction}

Colloidal crystals have been attracting much attention as promising photonic crystals. In particular, silicon inverse opal of a close-packed face-centered cubic (FCC) colloidal crystal is known to show three-dimensional (3D) perfect photonic bandgaps [1]. If sufficiently large and high-quality colloidal crystals can be fabricated, they realize the application of colloidal crystal to a 3D optical integrated circuit or optical computer in the future. To fabricate larger colloidal crystals, bottom-up-type self-assembly (crystallization) methods become much more advantageous than other top-down-type micro-fabrication methods [2].

Many studies have so far reported various methods of colloidal crystallization, such as 
convection-assisted dip coating methods [3], template-assisted sedimentation methods [4], shear-assisted alignment methods [5, 6], unidirectional growth methods driven by diffusion of pyridine [7], centrifugal sedimentation methods [8], etc. To grow close-packed colloidal crystals that have large size and high quality, high controllability is required. As such colloidal crystallization methods, we came up with the utilization of unidirectional growth of ice crystals, since many in-situ observation studies on the unidirectional growth of ice-water interfaces have already been performed using various sophisticated and highly-controllable apparatuses [9-12].

Regarding colloidal crystallization using freezing, there exists only one report as far as we know. Im et al. quenched water suspensions of polystyrene particles with liquid nitrogen, and obtained iridescent 3D colloidal crystals [13]. Although they clearly demonstrated that freezing is useful for fabricating colloidal crystals, they did neither control nor observe the crystallization processes. Thus, one has to clarify precise mechanisms of condensation and colloidal crystallization of particles during the freezing processes.

To achieve colloidal crystallization using unidirectional freezing, colloidal particles have to be rejected by growing ice-water interfaces. Uhlmann et al. studied the freezing of three liquid suspensions of ten foreign particles [14]. They clearly indicated that the rejection of the particles occurred when the velocity of an advancing solid-liquid interface was slower than a critical value. Hence from this result, we expected that freezing of colloidal random suspensions can become a promising means for colloidal crystallization.

In this study, we observed unidirectional freezing of colloidal crystals and colloidal random suspensions of polystyrene particles. Then we demonstrated the possibility of the 
unidirectional freezing as a novel highly-controllable method for colloidal crystallization.

\section{Experimental}

\subsection{Sample preparation}

Suspensions of polystyrene microspheres of diameter $d=200 \mathrm{~nm}$ in water were purchased from Duke Scientific Corporation (catalog No. 5020A). Volume fraction of the suspension $\varphi$ was 0.1 .

To confirm the rejection of polystyrene particles from ice-water interfaces, colloidal crystals were prepared by deionizing the suspensions. To deionize the suspensions, ion-exchange resin beads (AG501-X8 (D), BioRad) were added into the suspensions, and the suspensions were gently stirred for more than 3 days until they showed iridescence all over the suspensions. Then the colloidal crystals thus obtained were used for unidirectional freezing experiments. In these experiments, interfaces between growing ice crystals and colloidal crystals were observed. Here note that ice crystals could be easily grown in the colloidal crystals, since the colloidal crystals we used in this study contained sufficient amount of water (90\% in volume at first).

Another series of unidirectional freezing experiments were also carried out using the suspensions without deionization (as purchased), to demonstrate colloidal crystallization at interfaces between growing ice crystals and water suspensions of polystyrene particles.

\subsection{Experimental apparatus}


A growth cell was composed of two glass slides $(76 \mathrm{~mm}$ long $\times 26 \mathrm{~mm}$ wide $\times 1 \mathrm{~mm}$ thick) as horizontal top and bottom walls, and of two nylon wires (diameter $\phi=0.090 \mathrm{~mm}$ ) as spacers in the longitudinal direction (Fig. 1). Interspaces between the slides were sealed with a silicone adhesive as schematically shown in Fig. 1. A water suspension of polystyrene particles was injected into the internal space of the cell. Then, both open ends of the space were sealed also with a silicone adhesive.

Unidirectional freezing experiments were performed in an apparatus schematically shown in Fig. 2. The design of the apparatus was originally provided by Hunt et al. [9], and modified by Nagashima and Furukawa [10].

A growth cell was located in the apparatus as schematically shown in Fig. 2(a). The temperature profile in the particle suspension (hatched region in Fig. 2(a)) in the cell is schematically shown in Fig. 2(b). The temperatures of the right and left sides of the cell were controlled at higher and lower temperatures $\left(T_{\mathrm{H}}\right.$ and $\left.T_{\mathrm{L}}\right)$ by using two copper blocks whose temperatures were controlled by Peltier thermoelements. An approximately linear temperature gradient was provided in the gap between the higher and lower temperature regions [10]. Fig. 2(c) shows a schematic drawing of growing ice crystals in the hatched region. When the cell slid in the direction of a white arrow with the velocity $V$, the temperature profile provided stationary growing ice interfaces. The interfaces were formed at the position whose temperature $T_{\text {front }}$ was slightly lower than the melting temperature $T_{\mathrm{m}}$ $=0.0{ }^{\circ} \mathrm{C}$. Unidirectional freezing processes were observed in situ by transmission and reflection bright-field optical microscopy. 


\subsection{Characterization of the concentration of particles}

To probe the rejection of particles from ice-water interfaces, changes in complementary colors [16-17] of colloidal crystals in front of the interfaces were observed. Colors of transmitted light passing through a growth cell perpendicular to the horizontal top and bottom walls are complementary to those of Bragg reflections from $\{111\}$ faces of FCC colloidal crystals in the cell, since the $\{111\}$ faces usually stack parallel to a flat wall. The wavelengths of the Bragg reflection $\lambda_{\text {Bragg }}$ depend on the lattice spacing between the $\{111\}$ faces $d\{111\}$; the complementary colors also depend on $d\{111\}$. Thus the change in a particle volume fraction $\varphi$ can be characterized from the change in complementary colors.

\section{Results and Discussion}

\subsection{Rejection of particles from growing ice crystals}

To achieve colloidal crystallization by unidirectional freezing of colloidal random suspensions, colloidal particles have to be rejected from growing ice crystals. Then, before performing colloidal crystallization using colloidal random suspensions, first we tried to confirm the rejection of particles from growing ice crystals. To visualize rejection of particles, we performed unidirectional growth of ice crystals in "colloidal crystals" (instead of colloidal random suspensions). 
We observed complementary colors of colloidal crystals in front of growing ice crystals using a transmission optical microscope (Fig. 3). Fig. 3(a) was taken 2 s after we started the growth of ice crystals at $T_{\mathrm{L}}=-5.1{ }^{\circ} \mathrm{C}$ and $T_{\mathrm{H}}=5.0{ }^{\circ} \mathrm{C}$. In Fig. 3(a), the leftmost white region corresponds to ice crystals, and the other colored region shows colloidal crystals. In our experimental conditions, transmittance of particle random suspensions was low $(0.3 \%$ at the wavelength $\lambda=500 \mathrm{~nm}$ ). Then particle random suspensions looked dark in a transmission image (as lately shown in Fig. 5 (a)). Hence the white color of the ice crystals indicates that the ice crystals did not include particle suspensions. Disappearance of complementary colors in the ice crystals also showed that the ice crystals did not include colloidal crystals. In contrast, color gradations shown in the colloidal crystals demonstrated that lattice parameters of the colloidal crystals gradually changed in the $\mathrm{x}$ direction, resulting from the rejection of particles from the ice crystals.

Figs. 3 (b) and (c) were taken 41 and 107 s after the onset of the growth, respectively. As time elapsed, the ice crystals grew significantly. In these figures, a cellular structure of the ice crystals showed that the driving force for the crystallization was relatively large. Note that the position of the gradations of complementary colors also moved into the right direction accompanied with the movements of growth fronts of the ice crystals. This result clearly demonstrates that the gradations of complementary colors were formed by the rejection of particles from growing ice crystals.

At the rightmost position of Fig. 3, the complementary color was green. With decreasing distance from the growth fronts of ice crystals, the complementary color gradually changed from green to blue and also to red. To interpret this change in the complementary color, we 
calculated the relations between $\lambda_{\mathrm{Bragg}}, \varphi$, and complementary colors of transmitted light, according to the references [6, 16-17]. The results are summarized in Fig. 4. In this calculation, we assumed that the $\{111\}$ faces of the colloidal crystals were always aligned parallel to the horizontal top and bottom walls of the cell, since the thickness of the growth cell is very thin $(<0.1 \mathrm{~mm})$ [18]. From the comparison between the changes in the colors shown in Figs. 3 and 4, we concluded that the lattice parameters of colloidal crystals decreased with decreasing the distance from the growth fronts of ice crystals because of the rejection of particles from the growing ice crystals.

At the nearest position of the growth front, the complementary color of the colloidal crystals looked orange (Fig. 3). According to the simplest estimation explained in Fig. 4, the orange color corresponds to the volume fraction of colloidal crystals $\varphi=0.84$. However, this value is higher than the maximum volume fraction $\varphi=0.74$, at which particles are closely packed. This discrepancy was probably due to the too simplified model, the difference in the color sensitivities of human eyes and a charge-coupled-device camera used in this study, etc. [16-17].

\subsection{Growth of colloidal crystals by unidirectional freezing of polystyrene particle random suspensions in water}

As confirmed in the section 3.1, the rejection of particles occurred at growing ice crystals. Then, next we observed unidirectional freezing of water suspensions of polystyrene particles $(\varphi=0.1)$, using a reflection and transmission microscope (Fig. 5). The 
unidirectional freezing was conducted at $T_{\mathrm{L}}=-5.0^{\circ} \mathrm{C}$ and $T_{\mathrm{H}}=5.0^{\circ} \mathrm{C}$. To obtain smoothly grown grains of polystyrene colloidal crystals, the driving force for crystallization should be sufficiently small. Then, in the following experiment, we continuously retreated the cell at the retreat velocity of $V=0.01 \mu \mathrm{ms}^{-1}$.

Figs. 5(a), (b), and (c) respectively show transmission, reflection, and mixed (both transmission and reflection at the same time) images taken $22 \mathrm{~min}$. after the onset of the retreat. These images were taken at the same focus, although positions of corresponding interfaces look slightly different owing to tilt angles of the interfaces and different contrasts of figures. Fig. 5(a) shows blight white ice crystals on the left side and a dark particle suspension on the right side, since transmission light passed through transparent ice crystals, and did not pass through the opaque particle random suspension, as described in the section 3.1. In contrast, Fig. 5(b) represents areas colored in green, which corresponds to the typical diffraction color of FCC colloidal crystals at the following conditions: (1) particle diameter $d=200 \mathrm{~nm},(2)$ volume fraction of the particles $\varphi \approx 0.5$ or more, and (3) the $\{111\}$ faces of the crystals are parallel to the horizontal bottom wall of the cell. As shown in Fig. 5(c), rightmost edges of the green areas were located on the right side of the ice-water interface, and the positions of the rightmost edges and ice-water interface were significantly separately-placed (about $40 \mu \mathrm{m}$ ). From these results, we concluded that the nucleation and growth of the colloidal crystals occurred in front of the ice-water interface when the volume fraction of the particles increased beyond a critical value, while the other parts of the particle suspension remained as the suspension in which particles were randomly dispersed. 
We observed the cell from underneath using an inverted-reflection microscope. Hence, from the images shown in Figs. 5 (a) and (b), a cross-sectional structure of the sample can be schematically drawn as Fig. 5(d). This is probably because density of water is higher than that of ice. During the growth of ice crystals, heavier water came down to the bottom glass plate, and lighter ice crystals tended to growth on the top glass plate. Such the downward growth of the ice crystals increased the volume fraction of the particles on the bottom, resulting in the colloidal crystallization on the bottom.

In Fig. 5(b), more than a half of the area, columnar grains in front of the ice-water interface show green color, since their $\{111\}$ faces are parallel to the horizontal bottom wall of the cell and also to the longitudinal growth direction of the grains, as described above. In contrast, in front of the ice-water interface, there are many portions that do not exhibit green color.

Such alignments of grains are explained well as the results of the shape of the cell and preferential growth of grains, as follows. First, many grains nucleate on the ice-water interface with their $\{111\}$ faces parallel to the interface, while also many grains nucleate with their $\{111\}$ faces parallel to the horizontal bottom wall which is perpendicular to the ice-water interface. The latter grains correspond to the areas shown in green in Fig. 5(b). In contrast, the $\{111\}$ faces of the former grains cannot align parallel to the horizontal bottom wall, since their $\{111\}$ planes have already aligned parallel to the ice-water interface. Thus, the colorless portions between the green columns shown in Fig. 5(b) probably indicate the colorless grains aligned their $\{111\}$ faces parallel to the ice-water interface. The diffraction wavelengths of high-index planes which are perpendicular to a $\{111\}$ face and the 
ice-water interface are out of visible light region in many cases. Second, the green grains which nucleated with their $\{111\}$ planes parallel to the horizontal bottom wall could grow easier and faster than the colorless grains because of their preferential alignments with the horizontal bottom wall. Thus, the widths of the green grains became gradually wider during the horizontal growth.

Figs. 5(b) and (c) indicate that the colloidal crystal showed a columnar shape, as in the case of the unidirectional growth of colloidal crystals by centrifugation [8]. The formation of the columnar grains should be also due to the nucleation of colloidal crystals in front of the ice-water interface and to the subsequent geometrical selection of growing grains as described above. Thus, the findings obtained in the colloidal crystallization by centrifugation strongly suggest that the further decrease in $V$ or initial particle concentration will result in the further suppression of nucleation, yielding the enlargement of grains. Intentional addition of surfactants in the suspension would be also useful to control the nucleation rates of grains in front of the ice-water interface or to modify the shape of the interface. We will perform such experiments in the near future.

Fortunately, the advancing ice-water interface did not destroy the columnar grains of colloidal crystals until the grains had grown up to $\approx 40 \mu \mathrm{m}$ in length, since the color of a columnar grain was uniform at the microscopic level. The ice-water interface probably advanced with keeping columnar grains undestroyed in front of the interface, since colloidal crystals in front of the interface easily move rightward during the advancement of the ice-water interface. The ice-water interface probably pushed the colloidal crystals and grew smoothly, since water easily passed through the interspace between particles in the 
grains. To prove this mechanism, we will have to perform the precise in-situ observation of colloidal crystallization processes, in the near future. In addition, we will also carry out the precise characterization of the quality of colloidal crystals in front of the ice-water interface.

\section{Conclusions}

In this paper, we explored the possibility of unidirectional freezing as a novel method for growing colloidal crystals. We observed in situ the unidirectional freezing processes of the colloidal crystals and colloidal random suspensions. Then from the changes in the complementary colors of the colloidal crystals, we confirmed that the unidirectionally moving ice-water interfaces reject and concentrate the particles. We could also prove that colloidal crystallization proceeds in front of the unidirectionally growing ice-water interfaces. These results strongly suggest that the unidirectional freezing can become a promising means for colloidal crystallization. In the near future, we will elaborate to grow colloidal crystals of large grain size utilizing the good controllability of the unidirectional freezing.

\section{Acknowledgements}

This work was supported by the Grant for Joint Research Program of the Institute of Low Temperature Science, Hokkaido University. This work was partially supported by Grants in Aid (No. 24656016 (Y. S.)) of Scientific Research of the Ministry of Education, Culture, Sports, and Technology Japan. 


\section{References}

[1] A. Blanco, E. Chomski, S. Grabtchak, M. Ibisate, S. John, S.W. Leonard, C. Lopez, F. Meseguer, H. Miguez, J.P. Mondia, G.A. Ozin, O. Toader, and H.M. van Diel, Large-scale synthesis of a silicon photonic crystal with a complete three-dimensional bandgap near 1.5 micrometres, Nature 405 (2000) 437-440.

[2] C. Lopez, Materials aspects of photonic crystals, Adv. Mater. 15 (2003) 1679-1704.

[3] Y.A. Vlasov, X.-Z. Bo, J.C. Sturm, D.J. Norris, On-chip natural assembly of silicon photonic bandgap crystals, Nature 414 (2001) 289-293.

[4] A. van Blaaderen, R. Ruel, P. Wiltzius, Template-directed colloidal crystallization, Nature 385 (1997) 321-324.

[5] R.M. Amos, J.G. Rarity, P.R. Tapster, T.J. Shepherd, S.C. Kitson, Fabrication of large-area face-centered-cubic hard-sphere colloidal crystals by shear alignment, Phys. Rev. E. 61 (2000) 2929-2935.

[6] T. Sawada, Y. Suzuki, A. Toyotama and N. Iyi, Quick fabrication of gigantic single-crystalline colloidal crystals for photonic crystal applications, Jpn. J. Appl. Phys., 40 (2001) L1226-L1228.

[7] J. Yamanaka, M. Murai, Y. Iwayama, M. Yonese, K. Ito, T. Sawada, One-directional crystal growth in charged colloidal silica dispersions driven by diffusion of base, J. Am. Chem. Soc. 126 (2004) 7156-7157.

[8] Y. Suzuki, T. Sawada and K. Tamura, Colloidal crystallization by a centrifugation 
method, J. Cryst. Growth 318 (2011) 780-783.

[9] J.D. Hunt, K.A. Jackson, H. Brown, Temperature gradient microscope stage suitable for freezing materials with melting points between -100 and $+200{ }^{\circ} \mathrm{C}$, Rev. Sci. Instrum. 37 (1966) 805-805.

[10] K. Nagashima and Y. Furukawa, Nonequilibrium effect of anisotropic interface kinetics on the directional growth of ice crystals, J. Cryst. Growth 171 (1997) 577-585.

[11] K. Nagashima and Y. Furukawa, Solute distribution in front of ice-water interface during directional growth of ice crystal and its relation to interfacial patterns, J. Phys. Chem. B 101 (1997) 6174-6176.

[12] K. Nagashima and Y. Furukawa, Time development of a solute diffusion field and morphological instability on a planar interface in the directional growth of ice crystals, J. Cryst. Growth 209 (2000) 167-174.

[13] S.H. Im and O.O. Park, Three-dimensional self-assembly by ice crystallization, Appl. Phys. Lett. 80 (2002) 4133-4135.

[14] D.R. Uhlmann, B. Chalmers, K.A. Jackson, Interaction between particles and a solid-liquid interface, J. Appl. Phys. 35 (1964) 2986-2993.

[15] S.S.L. Peppin, J.S. Wettlaufer, M.G. PWorster, Experimental verification of morphological instability in freezing aqueous colloidal suspensions, Phys. Rev. Lett. 100 (2008) 238301-1-4.

[16] F. Birren, Color Dimensions, The Crimson, New York, 1934.

[17] E. Pretsch, P. Buhlmann, C. Affolter, Sturucture determination of organic compounds : Tables of spectral data, Springer-Verlag, Berlin Heidelberg, 2000. 
[18] T. Kanai, T. Sawada, J. Yamanaka, K. Kitamura, Critical concentration for colloidal crystallization determined with microliter centrifuged suspensions, Langmuir 21 (2005) 7633-7637.

\section{Figure Captions}

Fig. 1. A schematic illustration of a growth cell. Two nylon wires were sandwiched between two glass slides and placed parallel in the longitudinal direction of the slides. A flat space was prepared by filling the interspaces between the glass slides with a silicone adhesive. A suspension of particles in water was injected into the flat space. Open ends of the space were also sealed with a silicone adhesive.

Fig. 2. Schematic illustrations of a unidirectional freezing system. (a) A growth cell and two temperature-controlled copper blocks. The cell was inserted in square slits of the two copper blocks, whose temperatures were controlled at $T_{\mathrm{H}}$ and $T_{\mathrm{L}}$ using Peltier thermoelements. A bold black arrow indicates the longitudinal direction $x$ of the cell. (b) A temperature profile in the $x$ direction of the hatched region (shown in (a)) in the growth cell. (c) A magnified illustration of ice crystals and a particle suspension in the hatched region shown in (a). A white arrow shows the sliding direction of the growth cell. A growth front of ice crystals was formed at the position whose temperature $T_{\text {front }}$ was slightly lower than the melting temperature $T_{\mathrm{m}}\left(0{ }^{\circ} \mathrm{C}\right.$ in this study), since melting point depression probably occurred owing to the condensation of rejected particles in front of the growth interface as in the case of bentonite particles [15]. 
Fig. 3. Transmission images of advancement of ice-water interfaces and subsequent change in the complementary colors of colloidal crystals in front of the interfaces. Figs. 3 (a), (b), and (c) were respectively taken 2, 41, and $107 \mathrm{~s}$ after the onset of the growth of ice crystals at $T_{\mathrm{L}}=-5.1^{\circ} \mathrm{C}$ and $T_{\mathrm{H}}=5.0^{\circ} \mathrm{C}$. Scale bar represents $200 \mu \mathrm{m}$.

Fig. 4. A schematic illustration of relationships between Bragg wavelengths $\lambda_{\text {Bragg, }}$ particle volume fraction $\varphi$, and complementary colors of transmitted light. Each $\varphi$ is calculated as described in Ref. [6] using the corresponding $\lambda_{\text {Bragg }}$ value and following values; diameter of particles $d=200 \mathrm{~nm}$, refractive index of water $n_{\mathrm{w}}=1.33$, and that of particle $n_{\mathrm{p}}=1.59$.

Fig. 5. Growth interfaces of ice crystals and the formation of colloidal crystals in front of the interfaces. (a) A transmission image (interfaces between ice crystals (left) and colloidal crystals (or random suspensions to some extent) (right) were shown), (b) a reflection image (interfaces between colloidal crystals (left) and colloidal random suspension (right) were shown) and (c) a mixed image taken at the same focus. Scale bar represents $100 \mu \mathrm{m}$. (d) A schematic illustration of the cross-section of the growth cell. 
Fig. 1.

Journal of Crystal Growth

Yoshihisa Suzuki

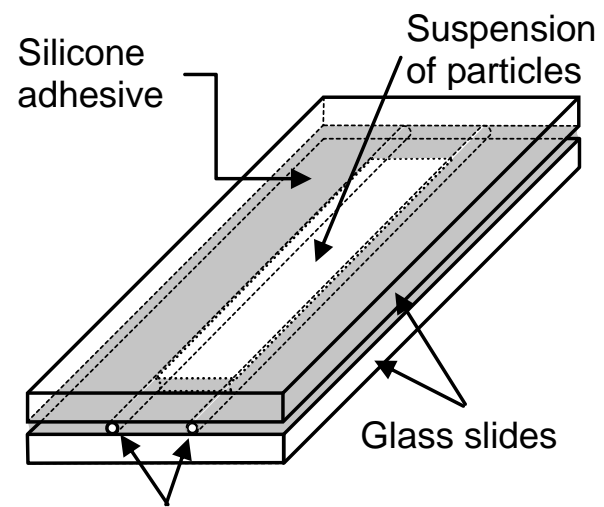

Nylon wires 
Fig. 2.

Journal of Crystal Growth

Yoshihisa Suzuki
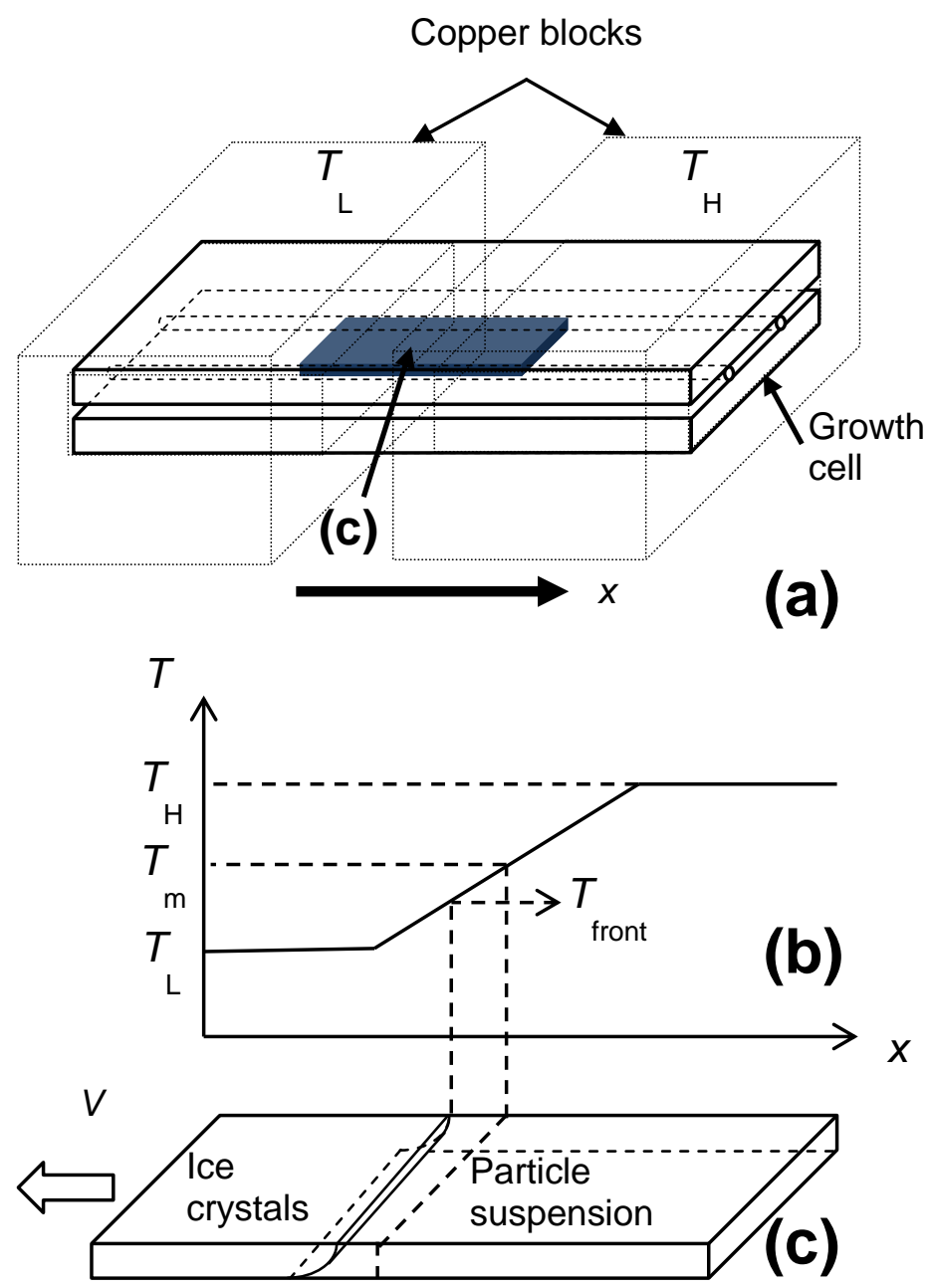
Fig. 3.
Journal of Crystal Growth
Yoshihisa Suzuki

Fig. 3.
Journal of Crystal Growth
Yoshihisa Suzuki

Fig. 3.
Journal of Crystal Growth
Yoshihisa Suzuki

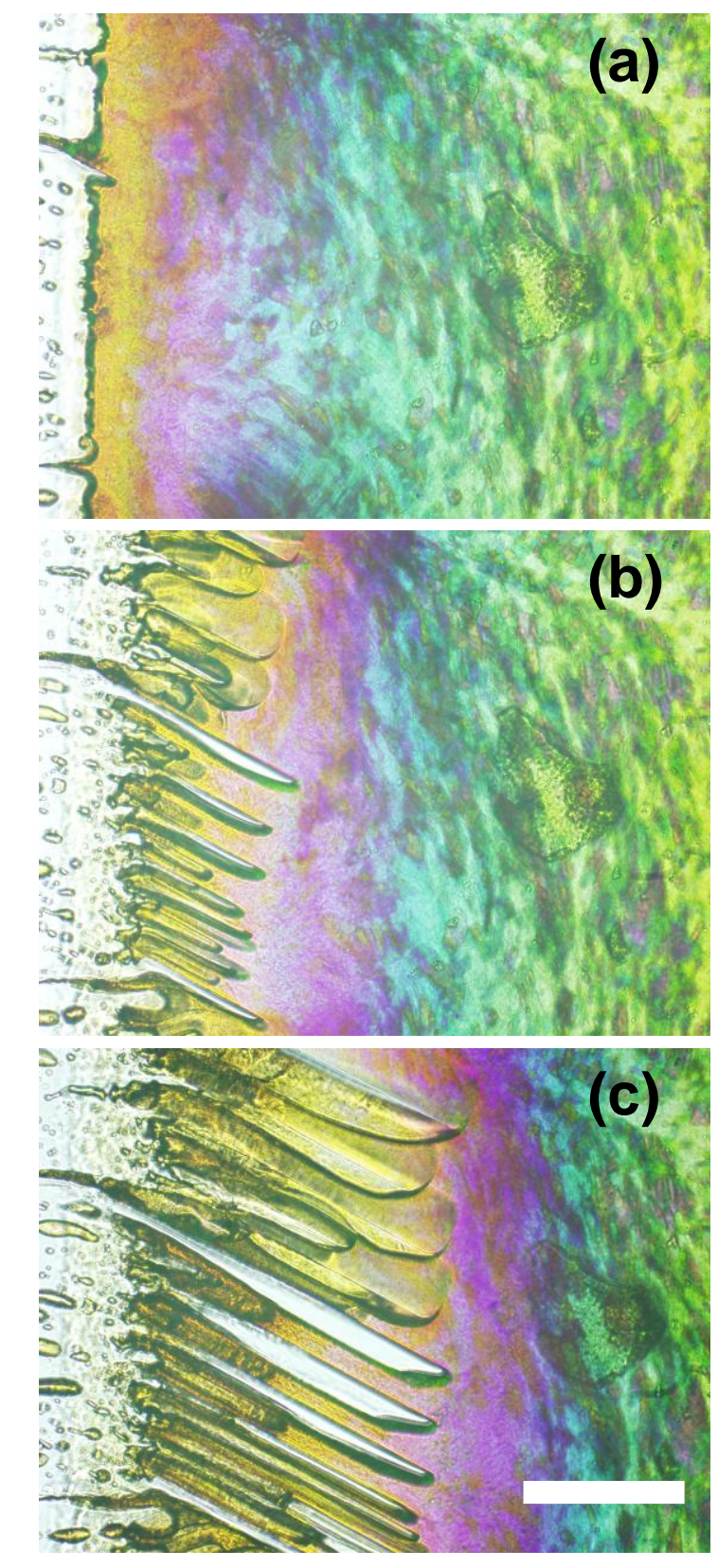


Fig. 4.

Journal of Crystal Growth Yoshihisa Suzuki

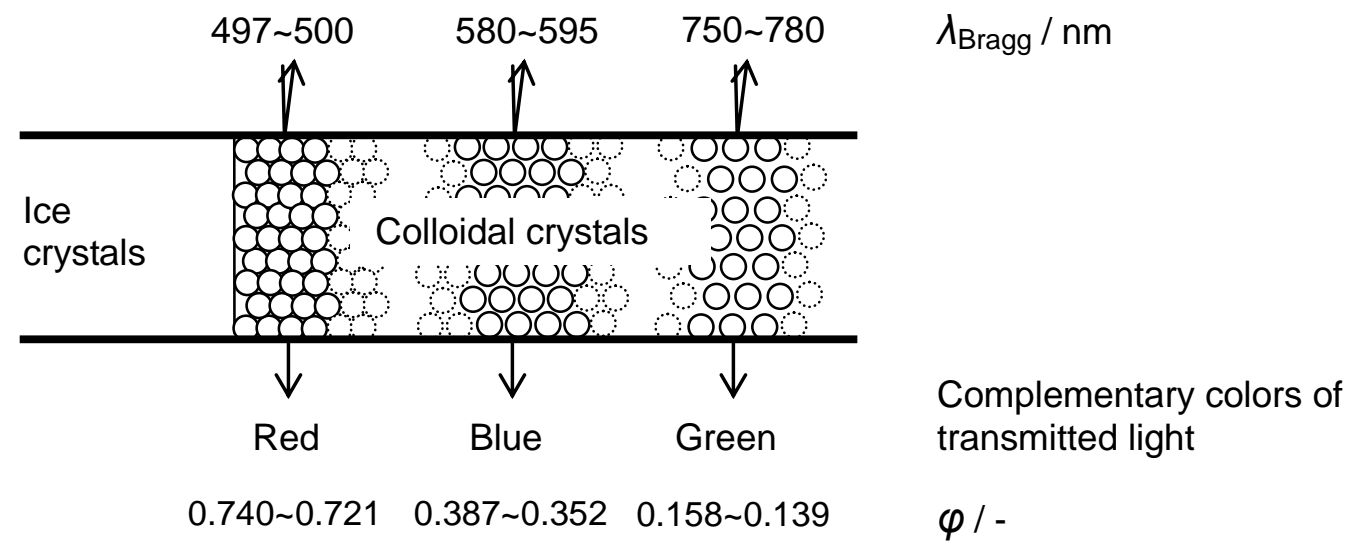


Fig. 5.

Journal of Crystal Growth Yoshihisa Suzuki
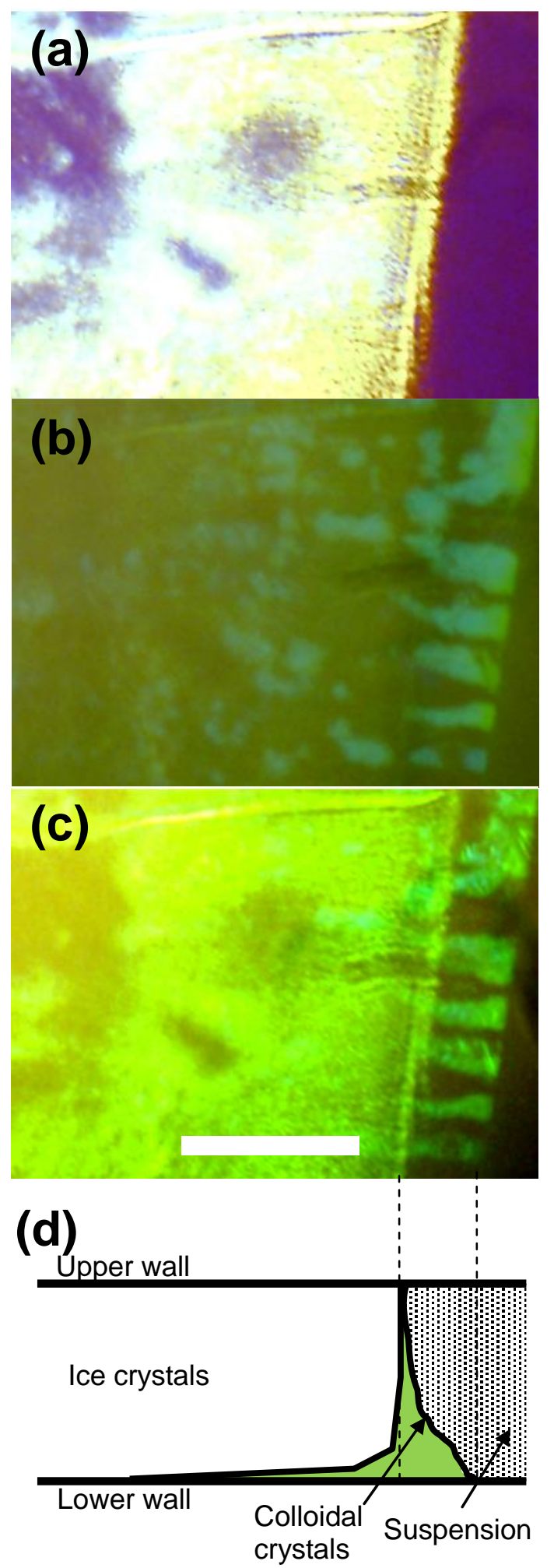